\title{
Morphologic Analysis of Multiple Tissues in Caveolin-1 Null Mice
}

Boyd $\mathrm{Qu}^{1,2 *}$ and Liping $\mathrm{Li}^{2}$

1. Department of Molecular Biology, Cell Biology \& Biochemistry (MCB), Warren Alpert Medical School of Brown University, Providence, RI, USA.

2. Center of Mineral Metabolism and Clinical Research, University of Texas Southwestern Medical Center, Dallas, TX, USA.

* Corresponding author: boyd_qu@brown.edu

Caveolin-1 is a principal structural protein of caveolae within the plasma membrane and has been implicated as a regulator of cellular signal transduction and systemic lipid and cholesterol homeostasis. $[1,2]$ Caveolin-1 null mice demonstrated that a loss of caveolae leads to a wide range of phenotypes including muscle, pulmonary and lipid disorders [3]. Caveolin-1 null mice showed dramatically reduced body weights, resistance to diet-induced obesity and altered metabolic and mitochondrial function in adipose tissue $[4,5]$. We screened a cohort of wild-type and Caveolin-1 null mice at 10-month-old with histochemistry (Figure 1) and described the morphological characteristics of multiple tissues in Caveolin1 null mice. The heart, liver, kidney, stomach, pancreas, skin and white adipose of Caveolin-1 null mice showed remarkable reduction in tissue cell size, cytoplasmic reduction, and increased intercellular spaces without a reduction in cell number. There was also significant thinning of the epidermis layer of skin. The brain, thymus, spleen, small and large intestines, testis, and ovaries did not exhibit significant differences between wild-type and Caveolin-1 null mice. Trichrome staining for collagen fibers exhibits fibrosis in the tissue matrix of Caveolin-1 null mice (Figure 2). Our results speculate that Caveolin-1 plays an important role in systemic lipid homeostasis in vivo. Further elucidation of Caveolin-1 function in these tissues may lead to novel targets for the relationship of Caveolin-1 with lipid transport and metabolism.

References:

[1] RG Parton, Annu. Rev. Cell Dev. Biol. 34 (2018), p. 111.

[2] P Liu et al., J. Biol. Chem. 277 (2002), p. 41295.

[3] M Drab, Science 293 (2001), p. 2449.

[4] B Razani et al., J. Biol. Chem. 277 (2002), p. 8635.

[5] IW Asterholm et al., Cell Metab. 15 (2012), p. 171. 
Cav-1 Wt
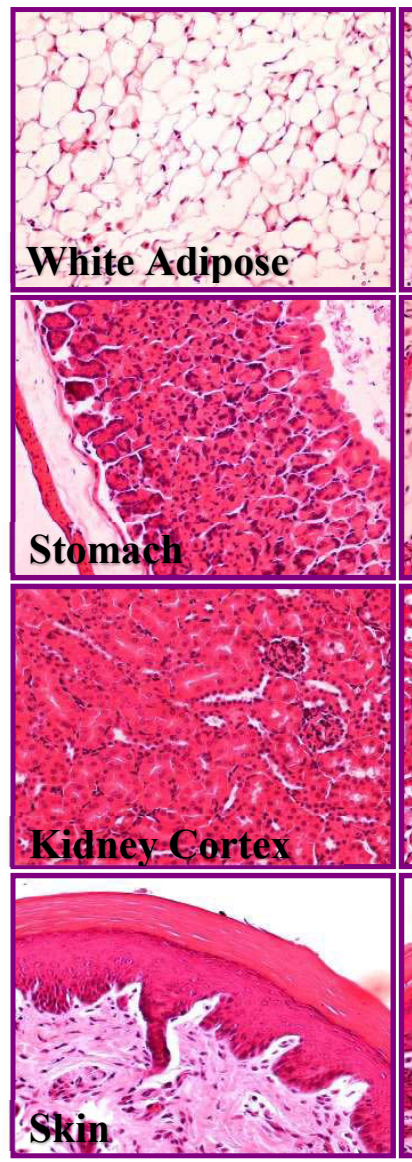

Cav-1 KO
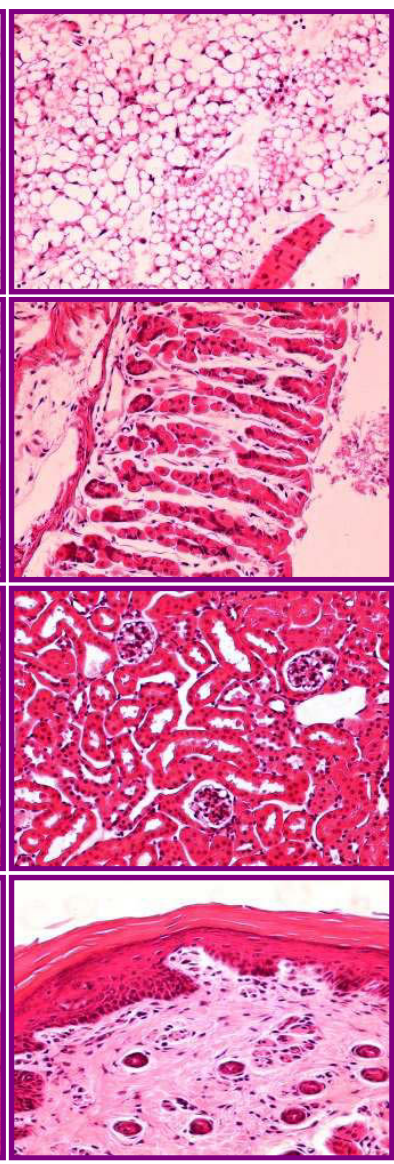

Cav-1 Wt
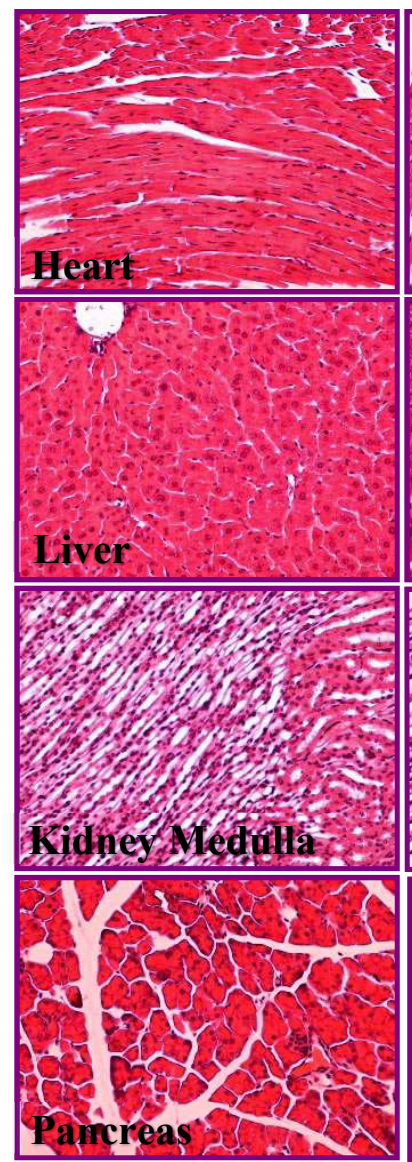

Cav-1 KO

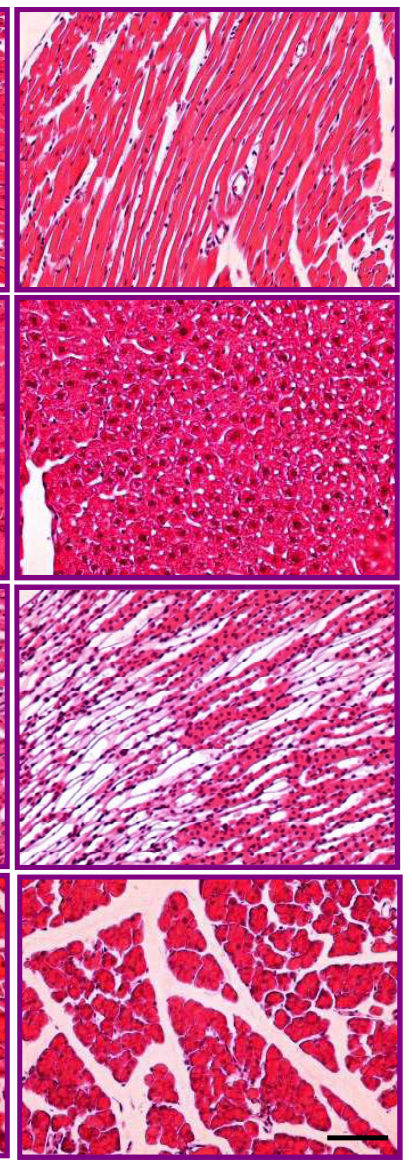

Figure 1. Morphological characteristics of multiple tissues in Caveolin-1 wild type (Cav-1 Wt) and Caveolin-1 null mice (Cav-1 KO) at 10-month-old. H\&E stained sections of multiple tissue from Cav-1 Wt and Cav-1 KO mice, by light microscopy. Note different type of cells from variety tissues present with smaller cell size, less cytoplasm and disordered arrangement as well as dilated intercellular spaces without a decrease in cell number. Bar $=100 \mu \mathrm{m}$

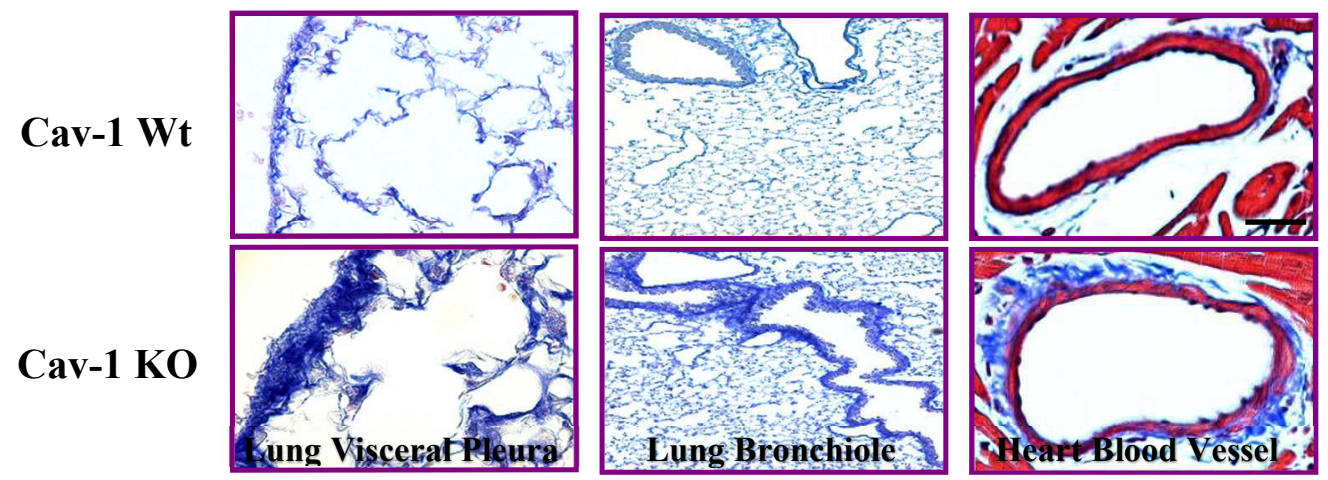

Figure 2. Trichrome staining of collagen within the tissues. Cav-1 Wt shows normal distribution of collagen fibers, stained blue. Cav-1 KO shows marked increase in collagen fibers around the serous membrane of lung visceral pleura, terminal bronchiole and blood vessels. Bar $=100 \mu \mathrm{m}$ 